\title{
Chapter 8 From Theories to Practicalities: Doing cross-cultural research fieldwork in Early Childhood Education \& Care (ECEC)
}

\author{
Yuwei Xu $\mathbf{u}^{1}$
}

The learning objectives of this chapter

- To bring theories of qualitative research methods into practicalities and real-life examples;

- To learn from empirical experiences of doing qualitative research in cross-cultural contexts;

- To exemplify how research questions may be finalized in qualitative research;

- To provide examples of practical challenges in qualitative research fieldwork and to share possible coping strategies;

- To transparent data collection processes in qualitative research and to showcase how research design can be refined, developed, and/or adjusted in such processes;

- To understand the researcher's roles and positionings in qualitative research.

Doing qualitative research in the field of social sciences can be both exciting and challenging. On the one hand, it enables researchers to look at issues that surround our social life in fresh and enlightening ways; on the other hand, naive qualitative scholars might find it sometimes 'tricky' when what they have read and planned does not go smoothly during their fieldwork in the 'realities'. Having enjoyed and suffered those processes in my own $\mathrm{PhD}$ journey, I would therefore be delighted to share here my experiences so far. It is hoped that other new researchers could be informed about possible practical difficulties that they might encounter. Based on my $\mathrm{PhD}$ research which looks at gender and its relevance regarding teacher-child interactions in Early Childhood Education and Care (ECEC) settings, this paper will first of all introduce the key motivations from which the research

\footnotetext{
${ }^{1}$ School of Education and Sociology, University of Portsmouth, UK; email: yuwei.xu@port.ac.uk
} 
questions were developed. It then moves on to discuss some practical concerns that research of this kind might encounter, such as ethical approvals, negotiating accesses, and so on. The paper will lastly conclude with an analysis of the researchers' own positions in the research and their respective impacts.

\subsection{Finalising research questions}

\subsubsection{Identifying research interests}

For most social sciences research, I believe that researchers' interested topics are usually inspired by our own life experiences - about ourselves and/or our surroundings. My personal experience suggests that a topic that is closely connected with my experiences has kept me motivated and enthusiastic throughout the $\mathrm{PhD}$ study. Being a man studying and working in the field of ECEC, I always found myself outnumbered by girls both in the Chinese and British contexts (I was one of the three boys out of 33 students on the B.Ed in Early Childhood Education program in a Chinese university, and the only male student on the MA Child Studies program in an English university). Having 'suffered' this paucity of men in both positive and negative ways, I became curious about why there are so few men in the field and am keen to find out the answers. This then led to my Master's dissertation in the UK and another small-scale study during my university work in China, both of which were relevant to male teachers in ECEC. It is those two studies that laid the foundation of my $\mathrm{PhD}$ project. I found it particularly exciting if to look at the issue of male participation in ECEC cross-culturally, and comparative study thus becomes central to my research design.

\subsubsection{Formulating research questions}

When your broad research interests are identified, it is then time to 'narrow down' and think about more concrete research aims and questions. For those of you who might be considering doing a PhD during Master's study, it is recommended that you conduct your Master's thesis as a pilot study for the PhD. In so doing, you will have chances to think deeper about your research plans. As I said, my Master's dissertation was also about male teachers in ECEC. I interviewed five males working in this area in regards to their experiences, and through these interviews I realised that it is not sufficient to just interview men teachers. Consequently, I further carried out a study that included both male and female teachers' perspectives 
about having men working in ECEC. Again, I found this time that observations and children's perspectives are necessary in better understanding whether men are needed or not.

Another advantage of doing some piloting is that you get to know about the literature. As you have already conducted systematic literature review for your Master's project, it significantly helps you become familiarized with the research field as well as identify research gaps for your PhD. It turned out that the gaps that I draw from literature matches perfectly with what I found useful from the piloting, and therefore, my $\mathrm{PhD}$ research aims are well justified. These consist of observing teacher-child interactions in ECEC classrooms, listening to teachers' and children's voices, and comparing different cultures, so as to examine whether or not men may make different contributions to ECEC. The research questions are based upon these aims and are further developed through development of theoretical framework and as research goes on.

\subsubsection{Refining research consistently}

Although you will need to have some fundamental questions when you start with your $\mathrm{PhD}$ research, the research questions are never finalised until the last minute. This process of consistent refinement of research questions usually starts with the development of theoretical framework, which covers your fundamental epistemology and ontology, as well as understandings of key concepts in your research. At this stage, you might find yourself submerged by terms such as poststructuralism, critical theory, structuralism, Marxism, and many other social theories; and perhaps the more you look into them, the more confused you could be. My experiences regarding this process was that, when I read around theories I often communicate and debate with other academics and non-academics to help reflect on myself. Do not just read the books and speak to your supervisors - you can discuss your thoughts with whoever might be interested in your research, and get them to ask you questions. To illustrate, as my theoretical framework focuses significantly on theories of gender, I often share my understandings of gender with my friends (researchers and non-researchers) and try to defend myself. By doing so, I gradually understand better about what I read in books and become critical about different theories. As your theoretical framework develops, you might want to rephrase and/or revise your research questions based on your new understandings. Be aware that this process will be continuous even during your fieldwork, your data analysis, and the writing-up. 


\subsection{From theories to practicalities}

When I have had a set of guiding research questions and have come up with an exciting fieldwork plan, I was so optimistic that I will do a 'great' piece of research and was enthusiastic about applying what I have planned into the reality. Nonetheless, the reality returned me with more difficulties and disappointments than I have ever expected. I started to realize at this stage that research will unavoidably be compromised by practicalities you might encounter. For me, resilience and reflectiveness are the two key qualities for surviving the fieldwork, which will be reflected in this section by examples of how I dealt with the difficulties. Although there will be no distinctive recipes for coping with research practicalities in social sciences research, I will go through here my lessons for others' references.

\subsubsection{Gaining ethical approval(s)}

This is the primary stage when you wish to begin your fieldwork that involves human beings, and can be considerably tricky particularly if you are doing research with vulnerable groups (i.e. young children) - so be prepared to be asked to revise the ethical application forms for as many times as one can expect. If possible, refer to some similar and successful applications from your institution and see how they paraphrase the languages and address the nuances. Also check the time schedule for submissions with your ethical committee as early as possible, to avoid holidays or 'peak period'. If you are doing research in local schools, make sure you are also aware of local policies regarding research access. To take my case as an example, I never knew that I also need to gain ethical approval from the city council in Glasgow in order to do fieldwork in local schools (neither me nor my two supervisors had research experiences in Scotland before). First of all, my original time schedule thus had to be deferred to allow time for this separate ethical application. But what was worse was that I then got declined to access local schools in Glasgow. According to feedback from the local government and to information from staff members in our university, there seemed to be a general conflict between educational research fieldwork and schools' intensive timetable in Glasgow and only studies that are deemed as directly 'beneficial' by the government will be allowed. This meant that for me to do my research in Glasgow became impossible even after some arguments and negotiations, and my research plan was significantly delayed. My strategy to solve this problem was to seek possibilities around nearby councils and I emailed universities in other Scottish cities for help. I also contacted some local communities and networks that are related to this research topic, to see if they can be of any help. Eventually, I was able to gain approval from Edinburgh City Council and finished my fieldwork there. 
In addition, if you are doing comparative studies in different parts of the world, you also need to learn in advance what kind of local ethical processes are required. To illustrate, in order to do my fieldwork in Hong Kong, I also submitted a third ethical application to the University of Hong Kong's ethical committee as a visiting research student, and adapted to local principles. Whereas in Mainland China, there are no such ethical approvals. In such cases when I did research in all these different cultures, I followed the main ethical principles at my home institution and meanwhile adapted to the differences in other cultures. Continuous reflectivity was conducted to reflect about if and how those general principles and specific adaptations might have influenced upon the research, and was then written into the methodology chapter.

Overall, in gaining your ethical approvals, a key message that I want to convey is to make sure you are aware of different policies in advance and anticipate possible difficulties that may delay your research - and most importantly, get prepared as early as you can so as to avoid disappointment in your intensive research schedule.

\subsubsection{Strategic recruitment}

After obtaining required ethical approvals, you are then allowed to contact potential participants and recruit them for your research. But do not wait until you have recruited all participants to start the fieldwork, and sometimes it is always wise to do some piloting as soon as you find one or more suitable participants. On the one hand, it saves you time while waiting for responses from other potential participants, and can help you develop your methods as you practice more. On the other hand, it is also a good strategy for recruitment itself. When you get to know people from the front lines, they might be able to help you expand your network and find other participants. For me, my time schedule was especially intensive due to the significant delay by ethical approvals, and I was doing fieldwork in three different cities across continents. Since my Scottish fieldwork was unexpectedly moved to Edinburgh, and I had no local connections there, I contacted a local organization that is related to my research topic to find me two participant schools. I then started my fieldwork immediately with these two schools, while still looking for other participants. It turned out that I was able to identify some other schools through the information provided by staff members in these two schools. This snowball sampling (Lewis-Beck, Bryman, \& Futing, 2004) was found particularly useful throughout my research, and I was able to recruit participants effectively in all three cultures.

If you have tried all your networks and still lack participants even after the 'snowballing', some other strategies that I used included calling as many schools as possible from the school lists on the government website, or going to any schools that you may come across and asking for access. These do not sound like perfect 
ways of recruiting, but it did turn out to be of some help as I recruited two schools in those ways. Just be polite and explain everything clearly, and it does not harm even if people do not respond to you.

\subsubsection{Approaching the participants}

Once you have survived from gaining ethical approvals and negotiating access to schools, you will come to a more challenging but exciting stage of the fieldwork - approaching the participants. As my research looks at teacher-child interactions and includes qualitative methods such as observations of classrooms, interviews with teachers, and pictorial conversations with children, I needed to get in touch with both teachers and children. Usually I was introduced to the participant teachers by the gatekeepers (the head teachers) after having had quick conversations with the latter. In some classrooms I was not introduced to the children by the teachers, and I found it might slightly be quicker for the children to approach me if I was properly introduced. With the majority of the teachers, they were warm-hearted at the very beginning and I easily felt welcomed. Sometimes the teachers were just too busy to look after me and I just made sure I went to them when they were less busy. It was also sensible to let the teachers know more about myself in order to build up the relationships. With the children, some of the 'brave' ones might come and talk to you to show their curiosity - but be patient, it can take from as fast as a couple of minutes to as long as a whole day or more. You can also try approach them first, but do not push it or get discouraged if the children feel reluctant to talk to you at the beginning. It also helped when some children approached you and interacted with you well - other children will see this and start to come close to you. More details from my experiences will be provided later in this chapter about doing research with young children.

\subsection{Conducting qualitative research in ECEC}

As mentioned earlier, my research included three main qualitative methods, namely observations, interviews, and pictorial activities with young children. I will now go through some of the practical concerns while carrying out those methods. 


\subsubsection{Observations in ECEC classrooms}

Motivated by the methodological gap in literature that few studies have done observations on how teachers' gender may impact upon their daily interactions with young children, I observed 17 early childhood classrooms altogether in Edinburgh, Hong Kong, and Tianjin City. The observation was more or less unstructured, though considerably informed by research objectives and the literature (Brody, 2014). It focused on the whole of a typical, coherent day in early childhood settings in the three cultures, so as to capture all possible interactions between the teachers and the children and representing the complexity and dynamics in ECEC. Usually in each setting, I may use one day to get myself familiarized with the daily routines, the organization of the classrooms, and the teachers and children; at the same time, some remarkable incidences that were relevant to the research topic were also noted down. Then there were a few more days of observations either focusing on the interactions between children and the male/female teacher, or both. Children's individual and group behaviours were sometimes recorded also, if regarded as relevant. In classrooms where there were more than two staff members (which were cases in all Scottish and Chinese settings), emphasize was put on the two participant workers for easier and in-depth data collection but the non-participating staff members' interactions with the children were sometimes written down when they were particularly interesting and were expected to be contributing to my research findings.

No videos or photographs about the interactions were taken in respect of ethical issues, hence the observation relied merely on my individual observation and note taking (in Chinese and Hong Kong kindergartens where taking pictures of children were regarded as less serious as in Scotland, I did take a few photos of their interactions under the permission of the teachers. But those photos were only used as reminders of memories, and all children's faces were avoided in the photos. I also took pictures of the environments in all settings of the three cities to assist with descriptions of the settings). The effectiveness of such a strategy was assured by observing for more than one day and the observations usually ceased when I felt that nothing really special had been observed that differed from previous days. In addition to written descriptions of observed behaviours and incidences, I also sought explanations of certain scenarios from the teachers (or sometimes from the children if they were able to illustrate), for well-informed data sources. The observation notes were further supplemented by daily fieldwork dairies that I wrote in the end of each fieldwork day, for summarizing the overall impressions and guiding later observations.

Specifically, I started with the intention to observe by distance but it turned out to be impossible with young children who are usually less independent, nor in early childhood settings that are often very busy and turbulent. Having realized this during my pilot studies, I then decided to become partially involved in the classroom life either actively or passively. This may consist of the following dimensions: 1 . as 
a participant in some of the activities, such as stories, singing, and outdoor activities, which allowed me to familiarize with the setting more quickly, to learn about the cultures in different settings, and most importantly, to gain trust from and build relationships with the children and the teachers; 2 . as an alternative to other staff members (especially when they were unavailable to all children) who sometimes helped with the organization of the classroom and to whom the children may turn for help. This, on the one hand, enabled the children to feel close to me; but on the other hand, it also gave the children an impression that I might be one of the 'disciplinarians' in the classroom. Although this kind of impression was not obvious enough to have influenced the research, I was aware that some children may have felt obligated to agree when I approached them for the pictorial activities. 3. as a 'researched' whose interactions with the children sometimes revealed gendered aspects that are well related to this research. Being a man who was unusual in early childhood settings myself, how the children responded to me in possibly gendered ways appeared to be worthwhile data for this project. At the same time, in minimising the negative impacts of my involvements in the researched environments, I paid particular attention not to interfere with how the teachers lead and organize the days, not to ever discipline the children, and to reduce the possible power distance through play with them.

\subsubsection{Interviews with ECEC practitioners}

I mentioned while describing the observation process that, explanations on certain observed interactions were sought from the practitioners. This made up part of the interviewing instruments and was conducted either formally or informally. Depending on each participant's time availability, I may talk to them informally throughout the day or find a separate time to discuss the incidences formally. It was rather flexible due to the teachers' busy schedules. Both ways worked well and no significant difference was noted. More importantly, a formal interview was also carried out with every participant teacher to explore their experiences and knowledge about working with young children, and their perspectives on gender and ECEC. A list of promoting questions and themes were prepared for a more purposeful interviewing in this regard, but space was allowed for opened-up answers and topics around the issue of gender and ECEC. The interview usually took around $0.5-1$ hour and took place in the participant's workplace. All interviews were recorded with the interviewees' consents. The interviews were supposed to be completed at the beginning of the fieldwork, for me to become relatively informed for the observations. However, and again, this was compromised by time realities and the interviews happened flexibly before, during, or after the observation periods. The planned attempt to contextualize through the interviews was not badly affected though, as my knowledge about the macro and micro contexts were obtained either through informal talks with head teachers, the 
participant teachers themselves, other staff members, and the observations, or were 'naturally' increased as my fieldwork experiences were enriched.

\subsubsection{Pictorial activities with young children}

For triangulated understandings of gender and ECEC, I also sought for children's perceptions of their teachers' gender in this research. Considering the difficulties and limitations of directly interviewing young children (Tisdall, Davis, \& Gallagher, 2009; Waller \& Bitou, 2011), I used pictures to promote children's expressions about their views. Three pictures were produced for the children's review, representing three types of adult behaviours that were common (or at least may happen) in early childhood settings and were culturally regarded as 'femaleoriented', 'male-oriented', or 'gender-neutral' respectively. The first picture involves a person carrying a child in his/her arms, the second is about someone kicking a ball, and lastly there is an adult reading a book (stories) in the third picture. All three persons are supposed to be presented with gender-ambiguous figures for the children to identify. For each picture, the children were primarily asked about what they saw, who they saw, and why; and further conversations were encouraged according to the children's responses. Gender were examined in their answers, and the pictures were specifically related to the children's teachers so that they were able to comment about their teachers' gender. Conversations with the children may last up to 10 minutes and were recorded with their own and parents' permissions. The pictorial activities usually happened after a few days' observations, when the children were getting familiarized and close to me.

Using pictorial activities to do research with young children turned out to be welcomed by the children, as many of them actively enquired to do the activities with me. Their perspectives about gender and their teachers were reflected in their answers, providing interesting and useful data for my research. Many of those answers coincided with data collected through observations and teachers' interviews too, hence are considered to be trustworthy. However, upon critical reflections I am also aware that the research design can be flawed in several ways. Firstly, the children's answers may be unstable. Although I had not been able to check the stability of all children's responses, I randomly 'tested' some of them by asking the same questions more than once and at different times (or sometimes it was the children themselves who came to me and offered to do the activities again). Some of their answers could be different from time to time, but not necessarily regarded as invalid. Children in the early childhood stage may have less established values and are still constructing their 'world', hence it is sensible for them to have fluid expressions. For instance, many teachers who participated in my research stated that, when a child said he/she likes a particular teacher, it does not mean that he/she does not like the other teachers. It may be that at a certain time and for a certain reason, the child likes that teacher 'more' than the rest. 
Secondly, the children's answers may be influenced by their peers. To reduce the uneasiness of one-to-one conversations possibly revealed by some children, I tried to do the activities with more than one child at the same time. But it turned out to be an inadequate strategy as the children would often repeat one another's words. Although this idea was later totally abandoned in my research, I have noted that even when having the conversation individually, the children's answers would somehow be influenced by others. For ethical considerations and in order to provide the children with a comfortable environment, all activities with children were conducted in the classrooms or around. As a consequence, the activities were therefore exposed to other children who liked to be around and give answers (this was particularly true for those who had already done the activities). In most cases the 'disturbing' children were asked to stay away either by the teachers or myself, so such impacts were indeed at minimum. Having said that, I would also acknowledge the peer influence on the construction of children's gender identities and world values (Frosh, Phoenix, \& Pattman, 2001).

Last but not least, the children's answers can be hampered by a number of factors. I already mentioned that some children may feel nervous during the one-toone conversation, probably because they had little experience of doing this kind of activity and especially when it was with a rather stranger. A further reason that may lead to children's uncomfortableness was the power distance perceived by them, and this was particularly true in Hong Kong and Mainland China. Although I tried my best to stand away from the power relations, I was sometimes inevitably involved in them. For example, the teachers in Chinese kindergartens may use me as a way of disciplining the children, by saying that: "if you don't behave, $\mathrm{Mr} \mathrm{Xu}$ will take you away"; or that "Mr Xu will write down your bad behaviours in his notebook". Most children would know that these were just jokes as they knew what I had been doing, but some may consequently be 'scared' of me. In addition, when I approached some children and they declined to do the activities with me, the teachers in both Mainland China and Hong Kong would then help me to make the request a second time (without being asked to). Even if not necessarily urging the children, the teachers' power as they practiced in everyday life may have impacted the request and therefore made some children feel obligated and pressured. In resolving these problems, I either explicitly told the children not to panic, that this was not a test and they were free to go if they did not want to do it; or used a few strategic measurements. To illustrate, I may hug the children or allow them to sit on my knees so that they can feel safe (with the presence of other teachers); I may play toys or chat about their families with them, to calm them down; and in case that some children might be afraid to say no, I observed their reactions all throughout the activities. Facial expressions, eye contacts, and body languages such as looking around or playing with their clothes, were all possible indications of their reluctance and/or nervousness. Once any of those were noted, I then used the two strategies just mentioned and they worked well with the majority of the children. With a few cases when the children kept feeling unwell, I stopped the research and let them go. 
The age group of the children, and their corresponding language ability, understanding level, and confidence level, can make some differences, too. These abilities may also vary from child to child. Where the selection of children participants' ages relied on the age groups that the male teachers recruited worked with, my research had to include children from as young as 2 years old up to the age of 6. Most children at the ages of 2-3 in my research were unable to orally express their views and some older children would also struggle to talk freely. With the anticipation that it was reasonable that not all children would get involved in the research, I tried my best to include as many as possible. And any information from the children, whether it was a yes or no answer, a name, or a long sentence, was treated equally and analysed properly in alliance with the research questions.

All in all, it is suggested that researchers need to take a reflective approach in minimising the disadvantages of chosen methods and in solving problems emerged during the research process. Some defects were unavoidable and were therefore made transparent in my writing, especially those embodied in research with young children. Those pitfalls also need to be well attended when analysing the research findings, so that no compromises were resulted from them in the study.

\subsection{The researcher and the research}

In addition to reflections on practical difficulties concerning the conduction of methods, I have also realized that my personal values and experiences may unavoidably have some influences on the research (Bryman, 2012). It is recognized that researcher's situation of himself/herself in the research, such as his/her gender, class, race, and other backgrounds, may sometimes have undeniable significance to studies of this kind (Ramanathan, 2005). By critically reflecting upon the research processes from question formulation, data collection, data analysis, and presentation of findings, I found it particularly worthwhile to point out the below factors on which my research might have been impacted by me as the researcher.

\subsubsection{The researcher's gender and the research}

Being a male who researches about gender in ECEC with teachers and young children, my presence appeared to be part of the 'gendered context' in this study. I already said at the beginning of this chapter that this project was inspired by my own experiences as a 'minority' man who studied and worked in early childhood settings in China and UK, and this has been frequently used as a 'shortcut' to build up rapport with the male teachers involved in my project. Some of them even said to me that I provided a role model to them, in terms of how they would pursue their 
career further. I believe such a relationship may have facilitated a good level of trustworthiness when the male teachers reflected about their experiences of working in ECEC to me. Nevertheless, it does not work the same way with the female teachers. Some psychological studies have noted that experimenters' gender may impact on participants' responses to issues like sex role attitudes in questionnaires (Galla, Frisone, Jeffrey, \& Gaer, 1981). Although such notation can hardly be applied to my study due to the totally different methodological nature, it encourages awareness of similar impacts. Williams and Heikes (1993) pointed out that, researcher's gender may make some differences in doing in-depth interviews with interviewees. The term 'social desirability bias' was used by them to describe 'the tendency of people to "adjust the truth" so that they sound nicer, richer, and more desirable to the researcher' (p.285) in those situations. I therefore suspect that it may be possible for the female teachers to 'adjust' their answers when they were interviewed about gender issues by a man (me), particularly for those female teachers in Chinese societies where gender relationships are still assumed to be hierarchical to a certain extent. Being aware of these possible impacts, however, I am confident that my personal capabilities in building up good relationships with females as evident from my daily life have helped to reduce them.

Additionally, my gender as a male might have impacted upon my relationships with children and ultimately the research, too. Primarily, my presence in the femaledominated environment may itself influence on the children's gender images, and even more when I interacted with them in their daily activities. Such influences are evident in some children's responses to the pictorial activities, as they sometimes pointed to/referred to me when asked about who are doing certain behaviours in the pictures. Moreover, I noticed that some children may feel reluctant to get close to me or to allow me to approach them, assumingly because of my gender. This on the one hand, could be resulted from their individual experiences with men outside the school settings, like some of the extreme cases in Edinburgh (that some children might have suffered or witnessed domestic violence by men at home); on the other hand, it might also be that the children were not having sufficient contacts with males other than their fathers at home and the usually only male teacher at school. Vice versa, there were also children who particularly liked me. Indeed, the popularity of my presence in all Chinese and Hong Kong kindergartens was massive, and was partly attributed to my gender according to the teachers. In reflecting upon all these responses that I experienced, I thus wonder that, had I been a female, would the children respond to and interact with me in different ways or not? Would it be easier or more difficult for the female researchers to establish rapport with some, if not all, of the children? The answers to these questions are to be explored in the future. In the meantime, I treated those children's responses and reactions to me as sources of data about their perceptions of gender, and these data were analysed and presented in the findings.

After all, being the only researcher in this project, I am unable to thoroughly reflect on the possible impacts of the researcher's gender. All I can reasonably say is that I am aware of the possibilities and have made available nuances transparent. 
I also admit that my research on its whole can be gendered in many ways. It is therefore up to the audiences and perhaps other female researchers who have done similar projects as I do to make further and critical judgments.

\subsubsection{The researcher's multiple identities and the research}

Although not as evident as gender, my other identities may have, to various extent, had some influence on my study. For example, the overwhelming popularity that I sensed in Chinese and Hong Kong kindergartens did not happen in any of the Edinburgh centres. But I am yet sure whether this was a cultural thing or whether it can be related to my identity as an Asian/Chinese. Similarly, being a Non-British and being a Chinese Mainlander may possibly have something to do with the interviews of teachers in the three cultures. Archer (2002) 's British Muslim participants in her study have revealed that, they tended to feel more comfortable with an Asian interviewer than with a British white one or others. It might also be the case that some of the teachers in my study may either feel easier or more cautious while reflecting about their experiences to me. Furthermore, I understood that being a PhD student might also be relevant. Some teachers from Hong Kong and Mainland China may sometimes ask me to comment on their practices in their daily teaching and caring, or seek confirmation from me for their answers to certain questions during the interviews. I was somehow seen as an 'expert' in this field of ECEC to them, which possibly put some pressure on them. Nevertheless, while acknowledging these nuances, I can hardly refer to any aspects of them that may have compromised my research considerably. I would also claim that, as a subjective human who has inevitably been involved throughout the research process, my gender, nationality and other individual characteristics, may have interwined with each other to add to the dynamics and complexities of the research process. To further refer to Archer (2002)'s study, when the Asian interviewer is a girl, she is even more welcomed by the interviewees. My multiple identities may have intersectionally made some differences in terms of how the participants react to me in different contexts and cultures, although not necessarily significant enough to impede the project.

\subsubsection{The researcher and the three cultures}

Lastly, the comparative nature of my study is significantly related to my own experiences with the three cultures, as well as my other intercultural experiences and skills. In comparative studies of this kind, familiarities of the researcher with the compared cultures are deemed to be importantly relevant (Philip \& Michele, 2014). I used the frameworks of Research circumstances and potential responses 
and Researcher skills and perspectives developed by Philip and Michele (2014) to argue how I am competent in conducting this study and how the study is thus not necessarily and/or considerably impaired. The Chinese culture is obviously my home culture, where I grew up and has been educated before postgraduate. I then studied in the UK (both England and Scotland) since 2010 and am increasingly getting familiarized with its cultures and education systems. Hong Kong is deemed to heritage from Chinese culture and to follow British education systems (Zhang, 1998), hence is a place that could be easily familiarized with by me. At the same time, all three contexts are also to some extent 'strange' to me. For instance, I have been away from China for a couple of years and am unfamiliar with the recent developments of ECEC there; it turned out that kindergartens in Tianjin have changed in many aspects and are quite different from what I used to have experienced about Chinese kindergartens. I do not live in Edinburgh and before I started my research fieldwork, I only paid a one-day visit to a nursery class in Glasgow. And I've even never been to Hong Kong until this project. Subsequently, I regard myself as in a good balance between 'making the familiar strange' and 'making the strange familiar', which appears to be a reasonable position in pursuing this study (Alexander, 2000; Philip \& Schweisfurth, 2014).

\subsection{Conclusion and recommendations}

To summarize, I have discussed in this chapter my experiences of doing qualitative research in three different cultures of Scotland, Hong Kong, and Mainland China, starting from the formulation of research questions and development of theoretical framework, ethical approvals and recruitments of participants, to practicalities of approaching the participants and carrying out various research methods. Some lessons were offered in terms of how to deal with unexpected issues in beginning the research fieldwork, including early attendances to ethical requirements, strategic sampling methods, and interactive communications with participants. Details of the research processes were also reflected towards their influences on the research, with particular focus on doing activities with young children. Observations and one-to-one interactions with the young children can be complicated and dynamic, and researchers should by all means prioritize children's individual needs over the research and be as sensitive as possible throughout the research fieldwork. There were analyses on the relevance of researcher's subjectivities to research in the last section, and aspects such as researcher's gender, ethnicity, and personal experiences were illustrated as more or less impacting on the research processes or results. To conclude this chapter, I would address the key words that have been central to my research journey so far, which are: motivation, resilience, adaptation, persistence, and last but not least, confidence.

Box 8.1 Suggested coping strategies from this chapter: 
- Combine your life experiences with your social sciences research, to keep yourself motivated and inspired;

- Speak out your thoughts and talk to people, to help structure your theoretical framework and to keep your mind critical;

- Get yourself familiarized with local ethical requirements as early as possible, to avoid possible delays in research fieldwork;

- Allow your participants chances to know about yourself, rather than merely one-way communications - it helps in building up the relationships;

- $\quad$ Patience is particularly vital when doing research with young children, and be tolerant with silence when you are talking to them;

- Finally, be prepared to encounter unexpected difficulties and be confident that you are able to cope with them sooner or later! 


\section{References}

Alexander, R. (2000). Culture and pedagogy: International comparisons in primary education. Oxford: Blackwell Publishers.

Archer, L. (2002) 'It's easier that you're a girl and that you're Asian': Interactions of 'race' and gender between researchers and participants. Feminist Review, 72, 108-132.

Brody, D. (2014). Men who teach young children: An international perspective. London: Institute of Education Press.

Bryman, A. (2012) Social research methods ( $4^{\text {th }}$ ed.). New York: Oxford University Press.

Frosh, S., Phoenix, A., \& Pattman, R. (2001). Young masculinities: Understanding boys in contemporary society. Hampshire: Palgrave Macmillan.

Galla, J.P., Frisone, J.D., Jeffrey, L.R., \& Gear, E.P. (1981) Effect of experimenter's gender on responses to a sex-role attitude questionnaire. Psychological Reports, 49, 935-940.

Lewis-Beck, M.S., Bryman, A., \& Futing, T. (2004). The Sage Encyclopedia of Social Science Research Methods (three volumes). Thousand Oaks, Calif.: Sage.

Phillips, D., \& Schweisfurth, M. (2014). Comparative and international education: An introduction to theory, method, and practice ( $2^{\text {nd }}$ ed.). London: Bloomsbury.

Ramanathan, V. (2005). Situating the researcher in research texts: Dilemmas, questions, ethics, new directions. Journal of Language, Identity \& Education, 4:4, 291-293. DOI: 10.1207/s15327701jlie0404_3

Tisdall, K., Davis, J., \& Gallagher, M. (2009). Research with children \& young people: Research design, methods and analysis. London: SAGE.

Waller, T., \& Bitou, A. (2011). Research with children: Three challenges for participatory research in early childhood. European Early Childhood Education Research Journal, 19:1, 5-20.

Williams, C.L., \& Heikes, E.J. (1993) The importance of researcher's gender in the in-depth interview: Evidence from two case studies of male nurses. Gender and Society, 7(2), 280-291.

Zhang, W. (1998). Young people and careers: School careers Guidance in Shanghai, Edinburgh and Hong Kong. Comparative Education Research Centre, The University of Hong Kong. 